\title{
Betatron radiation from density tailored plasmas
}

\author{
K. Ta Phuoc, ${ }^{1}$ E. Esarey, ${ }^{2,3}$ V. Leurent, ${ }^{2}$ E. Cormier-Michel,${ }^{2,3}$ \\ C.G.R. Geddes, ${ }^{2}$ C.B. Schroeder, ${ }^{2}$ A. Rousse, ${ }^{1}$ and W.P. Leemans ${ }^{2,3}$ \\ ${ }^{1}$ Laboratoire d'Optique Appliquée, ENSTA, \\ CNRS UMR7639, Ecole Polytechnique, \\ Chemin de la Hunière,91761 Palaiseau, France \\ ${ }^{2}$ Lawrence Berkeley National Laboratory, Berkeley, California 94720, USA \\ ${ }^{3}$ Department of Physics, University of Nevada, Reno, Nevada 89507, USA
}

(Dated: July 22, 2009)

\begin{abstract}
In laser wakefield accelerators, electron motion is driven by intense forces that depend on the plasma density. Transverse oscillations in the accelerated electron orbits produce betatron radiation. The electron motion and the resulting betatron radiation spectrum can therefore be controlled by shaping the plasma density along the orbit of the electrons. Here, a method based on the use of a plasma with a longitudinal density modulation (density depletion or step) is proposed to increase the transverse oscillation amplitude and the energy of the electrons accelerated in a wakefield cavity. For fixed laser parameters, by appropriately tailoring the plasma profile, the betatron radiation emitted by theses electrons is significantly increased in both flux and energy.
\end{abstract}




\section{INTRODUCTION}

Laser-driven, plasma-based accelerators are promising candidates for compact electron accelerators [1-3]. In a laser wakefield accelerator (LWFA), an intense laser pulse drives a large amplitude wave (wakefield) in a plasma with an ultrahigh electric field $(10-100 \mathrm{GV} / \mathrm{m}$ ) suitable for accelerating electrons to relativistic energies. At ultrahigh intensities, the laser pulse expels all of the plasma electrons from the region of the axis, forming a highly nonlinear wake consisting of an ion cavity (also referred to as the bubble or blow-out regime)[4-6]. Electrons from the background plasma can become trapped in this cavity and accelerated to high energies. Experiments on LWFAs have demonstrated the production of high quality electron bunches at the $100 \mathrm{MeV}[7-10]$ and $1 \mathrm{GeV}$ [11] levels over mm and cm scale plasmas, respectively. Such high quality electron bunches can subsequently generate x-rays through a variety of mechanisms [12], including betatron radiation [13-22], Thomson scattering [18], and synchrotron and free electron laser radiation [23] by passing the electron bunch through an undulator magnet. Since both the electron bunch and x-ray pulse are ultrashort (fs duration) and intrinsically synchronized to the drive laser pulse, such sources are ideal for use in pump-probe experiments for ultrafast science.

Betatron radiation [13-22] is emitted by an electron bunch accelerated in a plasmabased accelerator as a result of the transverse focusing fields of the wake. These focusing fields induce transverse oscillations (referred to as betatron motion) in the orbits of the accelerated electrons. The wavelength of this oscillation is the betatron wavelength $\lambda_{\beta}$, which for a cavitated wake is given by $\lambda_{\beta} \simeq(2 \gamma)^{1 / 2} \lambda_{p}$, where $\gamma$ is the relativistic factor of the accelerated electron and $\lambda_{p}=2 \pi c / \omega_{p}$ is the plasma wavelength, with $\omega_{p}=\left(4 \pi e^{2} n / m\right)^{1 / 2}$ the plasma frequency and $n$ the plasma density. Consequently, this betatron motion leads to the emission of synchrotron radiation (referred to as betatron radiation). For a small amplitude betatron oscillation, this radiation is emitted at a frequency $\omega \simeq 2 \gamma^{2} \omega_{\beta}$, where $\omega_{\beta}=2 \pi c / \lambda_{\beta}$ is the betatron frequency. For example, a $100 \mathrm{MeV}$ electron $(\gamma \simeq 200)$ undergoing a small amplitude betatron oscillation in a plasma of density $n=2 \times 10^{19} \mathrm{~cm}^{-3}$ $\left(\lambda_{p} \simeq 7.3 \mu \mathrm{m}\right.$ and $\left.\lambda_{\beta} \simeq 150 \mu \mathrm{m}\right)$ will emit betatron radiation at an energy $\hbar \omega \simeq 660 \mathrm{eV}$. This radiation will be confined to a cone angle of $\theta \sim 1 / \gamma$. Betatron radiation has been observed from plasma-based accelerators with both electron beam drivers [14, 20] and laser drivers $[17,21,22]$. 
Two important parameters [13] that characterize the features of betatron radiation are the betatron strength parameter $a_{\beta}$ (analogous to the undulator strength parameter in a conventional synchrotron) and the critical frequency $\omega_{c}$. In practical units, these can be written as

$$
\begin{gathered}
a_{\beta} \simeq 1.3 \times 10^{-10}\left(\gamma n\left[\mathrm{~cm}^{-3}\right]\right)^{1 / 2} r_{\beta}[\mu \mathrm{m}], \\
\hbar \omega_{c}[\mathrm{keV}] \simeq 1.1 \times 10^{-23} \gamma^{2} n\left[\mathrm{~cm}^{-3}\right] r_{\beta}[\mu \mathrm{m}],
\end{gathered}
$$

where $r_{\beta}$ is the amplitude of the betatron orbit. For $a_{\beta} \ll 1$, e.g., for a small amplitude betatron oscillation, betatron radiation is emitted at the fundamental frequency $\omega \simeq 2 \gamma^{2} \omega_{\beta}$. However, for $a_{\beta}>1$ betatron radiation is emitted within a broadband spectrum characterized by the critical frequency $\omega_{c}$ beyond which the radiation intensity rapidly diminishes. The radiation is emitted in the direction of the electron velocity within a vertical angle (normal to the plane containing the betatron orbit) of $\theta_{v}[\mathrm{rad}] \simeq 1 / \gamma$ and a horizontal angle (within the plane of the orbit) of $\theta_{h}[\mathrm{rad}] \simeq a_{\beta} / \gamma$. In the typical parameter regime of current laser-plasma experiments, $a_{\beta} \gtrsim 10$ and the betatron radiation consists on a femtosecond flash of broadband x-ray radiation extending up to the $10 \mathrm{keV}$ range, collimated within a few tens of milliradians. As an example, the parameters $\gamma=200, n=2 \times 10^{19} \mathrm{~cm}^{-3}$, and $r_{\beta}=2 \mu \mathrm{m}$ give $a_{\beta} \simeq 16$ and $\hbar \omega_{c} \simeq 18 \mathrm{keV}$.

To increase the photon energy of the betatron radiation, it is necessary to increase the critical frequency $\omega_{c}$ via increasing $\gamma, n$, and/or $r_{\beta}$. One straightforward method for increasing $\omega_{c}$ is to increase the electron energy $\gamma$. Although in principle this can be accomplished by using higher power lasers in longer plasmas, this approach may not be practical, since experiments are typically limited by the maximum available power of the laser system. Furthermore, using a cavitated wake in an initially longitudinally uniform plasma is inefficient for the production of betatron radiation for two reasons. First, the transverse amplitude of the electrons orbits in the ion cavity is of the order of 1 micron [21], whereas the diameter of the ion cavity is of the order of the laser spot size in the plasma, which can be several microns. Second, the electrons outrun the wake after the dephasing length [1] and are decelerated, whereas the laser pulse and ion cavity can propagate over a longer distance.

In this article we propose methods based on tailoring the longitudinal profile of the plasma density to shift the spectrum of the betatron radiation to higher energies. To approaches are investigated. In the first method, a region of depleted density is introduced in an 
otherwise initially uniform plasma. Accelerated electrons that enter the depleted region at the proper phase in their betatron oscillation will increase their transverse displacement, thus exiting the depleted region with a larger betatron amplitude. In addition, an increased betatron amplitude also implies that the axial electron velocity is lowered, which increases the dephasing length and the electron energy. Thus, a region of depleted density can lead to increases in both $r_{\beta}$ and $\gamma$, thereby increasing $\omega_{c}$. In the second method, a longitudinal step density profile is used wherein the density in the latter portion of the plasma is increased. The increased density decreases the radius of the cavitated wake (i.e., decreases the plasma wavelength), which increases the dephasing length and the electron energy gain [24, 25]. Thus, a step function density profile can increase both $n$ and $\gamma$, thereby increasing $\omega_{c}$. Numerical examples based on test particle orbits will be presented that indicate that the betatron radiation spectrum can be shifted from the $10 \mathrm{keV}$ range toward the $100 \mathrm{keV}$ range without increasing the drive laser pulse energy.

The remainder of this paper is organized as follows. Some of the basic properties of betatron radiation are reviewed in Sec. II. Modifications to the electron orbits as a result of modifying the longitudinal density profile with either a depletion region or a density step are presented in Sec. III. These results are obtained from numerical studying the motion of test electrons in analytically specified wakefields in the cavitated (blow-out) regime. The betatron spectrum generated from the orbits in the the modified density profiles are presented in Sec. IV for both single electrons and for a collection of test particles in an electron bunch. A discussion is presented in Sec. V.

\section{BASIC PROPERTIES OF BETATRON RADIATION}

An electron undergoing betatron oscillations in a plasma focusing channel, such as that produced in a laser wakefield accelerator, will emit synchrotron radiation. In the absence of an accelerating field, properties of the betatron radiation have been calculated in detail [13]. Here, we summarize the properties of betatron radiation from a single electron oscillating in the focusing fields of a plasma channel in the blowout regime. In the blowout regime, in which all of the plasma electrons have been expelled from the region near the propagation axis, the transverse force on a highly relativistic electron is described by an effective radial electric field given by $E_{r}=\left(k_{p}^{2} r / 2\right) E_{0}$, where $k_{p}=\omega_{p} / c$ and $E_{0}=k_{p} m c^{2} / e$. A highly 
relativistic electron will then execute transverse betatron oscillations in the $(x, z)$ plane given by $x(t) \simeq r_{\beta} \sin \left(k_{\beta} c t\right)$ with a transverse velocity $v_{x} \simeq c k_{\beta} r_{\beta} \cos \left(k_{\beta} c t\right)$, where $k_{\beta}=$ $k_{p} /\left(2 \gamma_{z 0}\right)^{1 / 2}$ is the betatron wavenumber in the blow-out regime, $r_{\beta}$ is the amplitude of the betatron orbit, $\gamma_{z 0}=\left(1+u_{z 0}^{2}\right)^{1 / 2}$, and $p_{z 0}=m c u_{z 0}$ is the axial momentum, which is constant in the absence of an axial accelerating field. Provided the number of betatron periods $N_{\beta}$ that the electron undergoes is large, $N_{\beta} \gg 1$, radiation is emitted in a series of harmonics and with frequencies centered about the resonant frequencies

$$
\omega_{N}=\frac{2 \gamma_{z 0}^{2} N c k_{\beta}}{\left(1+a_{\beta}^{2} / 2+\gamma_{z 0}^{2} \theta^{2}\right)},
$$

where

$$
a_{\beta}=\gamma_{z 0} k_{\beta} r_{\beta}
$$

is the betatron strength parameter, $N$ is the harmonic number and $\theta$ the observation angle with respect to the propagation axis, assuming $\gamma_{z 0}^{2} \gg 1, \theta^{2} \ll 1$, and $\left(1+a_{\beta}^{2} / 2\right) / \gamma_{z 0}^{2} \ll 1$. The intrinsic frequency width $\Delta \omega_{N}$ about $\omega_{N}$ of the radiation emitted along the axis $(\theta=0)$ is given by $\Delta \omega_{N} / \omega_{N}=\left(N N_{\beta}\right)^{-1}$. Radiation with frequencies in $\Delta \omega_{N}$ about $\omega_{N}$ are confined to a cone angle $\Delta \theta$ given by $\Delta \theta_{I}^{2} \simeq\left(1+a_{\beta}^{2} / 2\right) /\left(\gamma_{z 0}^{2} N N_{\beta}\right)$.

An important parameter characterizing the properties of the betatron radiation is the betatron strength parameter, $a_{\beta}$. For $a_{\beta}^{2} \ll 1$, the emitted radiation will be narrowly peaked about the fundamental resonant frequency, $\omega_{1}(N=1)$. As $a_{\beta}$ approaches unity, emitted radiation will appear at harmonics of the resonant frequency as well, $\omega_{N}=N \omega_{1}$. When $a_{\beta} \gg 1$, high harmonic $(N \gg 1)$ radiation is generated and the resulting synchrotron radiation spectrum consists of many closely spaced harmonics. Hence, in the asymptotic limit, i.e., $a_{\beta} \gg 1$, the gross spectrum appears broadband, and a continuum of radiation is generated that extends out to a critical frequency, $\omega_{c}$, beyond which the radiation intensity diminishes. In particular, the asymptotic $\left(a_{\beta}^{2} \gg 1\right)$ spectrum along the axis $(\theta=0)$ is given by

$$
\frac{d^{2} I(0)}{d \hbar \omega d \Omega} \simeq\left(6 / \pi^{2}\right) \alpha_{f} N_{\beta} \gamma_{z 0}^{2} \xi^{2} K_{2 / 3}^{2}(\xi)
$$

where $d^{2} I / d \omega d \Omega$ is the energy radiated per unit frequency $(d \omega)$ and per unit solid angle $(d \Omega), \alpha_{f}=1 / 137, K_{2 / 3}$ is a modified Bessel function with argument $\xi=\omega / \omega_{c}$, and

$$
\omega_{c} \simeq 3 a_{\beta} \gamma_{z 0}^{2} c k_{\beta}=3 c r_{\beta} k_{\beta}^{2} \gamma_{z 0}^{3}
$$


is the critical frequency (corresponding to a critical harmonic number of $n_{c}=3 a_{\beta}^{3} / 4$ ). The function $Y(\xi)=\xi^{2} K_{2 / 3}^{2}(\xi)$ is maximum at $\xi=1 / 2$ and decreases rapidly for $\xi>1$. Half the total power is radiated at frequencies $\omega<\omega_{c} / 2$ and half at $\omega>\omega_{c} / 2$. For frequencies $\omega \ll \omega_{c}$, the radiation intensity increases as $\left(\omega / \omega_{0}\right)^{2 / 3}$, and for $\omega \gg \omega_{c}$, the radiation intensity decreases exponentially as $\exp \left(-2 \omega / \omega_{c}\right)$. Furthermore, the average angular spread for the frequency integrated spectrum in the vertical direction (normal to plane of containing the betatron orbit) is $\theta_{v} \sim 1 / \gamma_{z 0}$, whereas in the horizontal direction (in the plane of containing the betatron orbit), $\theta_{h} \sim a_{\beta} / \gamma_{z 0}$. In particular, in the vertical plane, integrating the radiation spectrum over the vertical angle $\theta_{v}$ yields

$$
\frac{d^{2} I(0)}{d \hbar \omega d \theta_{h}} \simeq \frac{2 \sqrt{3}}{\pi} \alpha_{f} N_{\beta} \gamma_{z 0} \frac{\omega}{\omega_{c}} \int_{2 \omega / \omega_{c}}^{\infty} d \xi K_{5 / 3}(\xi)
$$

A highly effective method for shifting the radiation spectrum to high photon energies is to increase the critical frequency, $\omega_{c}$. In the blow-out regime,

$$
\omega_{c}=(3 / 2) c r_{\beta} k_{p}^{2} \gamma_{z 0}^{2} \sim r_{\beta} n_{0} \gamma_{z 0}^{2}
$$

The critical frequency, and hence the photon energy of the betatron radiation, can be increased by increasing the betatron orbit amplitude $r_{b}$, the plasma density $n_{0}$, and/or the electron energy $\gamma_{z 0}$.

The above results neglect the effects of an axial accelerating field. With acceleration, the axial electron momentum $p_{z}$ will increase. Assuming that $p_{z}$ varies slowly over a betatron period, the local betatron wavenumber is $k_{\beta} \simeq k_{p} /(2 \gamma)^{1 / 2}$ and varies slowly via $\gamma$, and the amplitude of the betatron oscillation is given by $r_{\beta} \simeq r_{\beta 0}\left(\gamma_{0} / \gamma\right)^{1 / 4}$, i.e., the orbit amplitude decreases as $\gamma$ increases, where $r_{\beta 0}$ and $\gamma_{0}$ are the initial values at the channel entrance [19]. Here the approximation $\gamma_{z}=\left(1+p_{z}^{2} / m^{2} c^{2}\right)^{1 / 2} \simeq \gamma$ has been made. As the electron accelerates, the critical frequency is shifted to higher values, $\omega_{c} \sim \gamma^{7 / 4}$ and the betatron spectrum broadens.

\section{ELECTRON MOTION IN A DENSITY TAILORED WAKEFIELD}

In a laser wakefield accelerator, the most straightforward method for enhancing the electron energy is to use higher laser powers and longer plasmas. However, this may not always be practical. Here, we consider two methods for enhancing the betatron radiation for fixed 
laser parameters, both of which rely on tailoring the plasma density profile. The first is to introduce zero density spatial gaps between uniform plasma density sections, so as to increase the betatron orbit of the electrons. The second is to introduce a higher density region behind a lower density region, so as to increase the electron energy. Both methods should increase the average critical frequency and thus shift the betatron radiation spectrum to high photon energies.

Here we consider electron motion within an idealized model of a laser wakefield accelerator in the blow-out regime [4-6]. A laser pulse with strength parameter $a_{0}>1$ propagates in an underdense plasma, expelling electrons from the region of the axis, creating an ion cavity and a highly nonlinear wake. The phase velocity of the wake is assumed to be equal to the laser group velocity in the plasma $v_{g}$. We assume the cavity to be a sphere [6] of radius $r_{b}=\left(2 \sqrt{a_{0}} / \pi\right) \lambda_{p}$, with the electromagnetic fields (axial $E_{z}$ and radial $E_{r}$ electric fields and an azimuthal $B_{\theta}$ magnetic field) of the wake within the blow-out region given by $E_{z} / E_{0}=k_{p} \zeta / 2, E_{r} / E_{0}=k_{p} r / 4$, and $B_{\theta} / E_{0}=-k_{p} r / 4$, where $\zeta=z+r_{b}-v_{g} t$ such that $\zeta=0$ and $r=0$ corresponds to the center of the blow-out sphere (cavity), $\zeta=r_{b}$ corresponds to the front of the cavity, and $\zeta=-r_{b}$ corresponds to the back of the cavity. The equation of motion for a test electron that is being accelerated inside the cavity is

$$
\frac{d \boldsymbol{p}}{d t}=\boldsymbol{F}_{\|}+\boldsymbol{F}_{\perp}=-\frac{m \omega_{p}^{2}}{2} \zeta \hat{\boldsymbol{z}}-\frac{m \omega_{p}^{2}}{2}(x \hat{\boldsymbol{x}}+y \hat{\boldsymbol{y}}),
$$

assuming $-r_{b} \leq(\zeta, x, y) \leq r_{b}$ (the test electron resides within the cavity), where $\boldsymbol{p}$ is the momentum and $(x, y, z)$ are the coordinates of the test electron. The electron is initially injected at the back of the cavity $z_{i}=-2 r_{b}$, with a transverse positions $x_{i}$ and $y_{i}$, and a velocity $\boldsymbol{v}_{i}$. Without lack of generality we assume $v_{y_{i}}=0$ and $y_{i}=0$, which restricts the test electron orbit to the $(x, z)$ plane. The first term, $\boldsymbol{F}_{\|}$, is responsible for the electron acceleration in the longitudinal direction $\hat{\boldsymbol{z}}$. As the electron becomes relativistic, its velocity becomes greater than $v_{g}$; the term $-\zeta=\left(v_{g} t-z-r_{b}\right)$ decreases and the accelerating force is reduced. The length for the electron to reach the middle $(\zeta=0)$ of the cavity and become decelerated $\left(v_{g} t-z>r_{b}\right)$ corresponds to the dephasing length $L_{d}$. The second term, $\vec{F}_{\perp}$, is the focusing force that creates transverse oscillating across the cavity axis at the betatron frequency $\omega_{\beta}=c k_{\beta} \simeq \omega_{p} / \sqrt{2 \gamma}$, assuming $\gamma_{z} \simeq \gamma$. Initially defined by the initial conditions at the injection into the cavity, the betatron amplitude $r_{\beta}$ decreases at the first stage of the electron acceleration. 
Since the forces in the cavity are directly dependent on the plasma density, an appropriate modulation of the density can be used to modify the relativistic electron orbit in such a way that the betatron radiation is shifted to high photon energies. Here, the methods proposed are based on a density modulation along the laser axis $\hat{\boldsymbol{z}}$. Axial modulations in the density can be formed either mechanically by guiding/blocking the gas flow [26] from a gas jet $\left(n_{1}=0\right)$, or by laser machining $\left(0<n_{1}<n_{0}\right.$ or oppositely $\left.n_{1}>n_{0}\right)$ [15, 16]. We will consider two specific cases (see Fig. 1): a finite density depletion and a density step.

For the first case (a density depleted region inserted between two regions of uniform density), betatron radiation can be enhanced as follows. In a density depleted region, the longitudinal and transverse forces are reduced (or eliminated in the case of zero density). An electron entering this depleted region will ballistically drift at constant energy and at a constant angle. If the length of the depleted region is sufficiently long, the transverse displacement of the electron entering the second region of uniform density can be greater than that in the first uniform region. Hence, the electron can enter the second uniform region with a larger betatron amplitude $r_{\beta}$ then before the depleted region. Since $r_{\beta}$ has increased, so will the critical frequency of the emitted betatron radiation, $\omega_{c} \sim r_{\beta}$. In addition, a larger betatron amplitude $r_{\beta}$ increases the transverse path the electron covers, which in effect decreases the axial electron velocity, i.e., $v_{z} \simeq v_{z 0}\left[1-\left(k_{\beta}^{2} r_{\beta}^{2} / 2\right) \cos ^{2} \omega_{b} t\right][13]$. This results in a longer dephasing length (the time for the electron to reach the middle of the cavity is longer), which increases the axial electron energy and consequently the critical frequency $\omega_{c} \sim \gamma^{7 / 4}$. The dephasing length $L_{d p}$ can be estimated by $L_{d p}\left(v_{z}-v_{g}\right) \simeq r_{b}$, where $v_{g}$ is the group velocity of the laser pulse, i.e., $v_{g} / c \simeq 1-\omega_{p}^{2} / 2 \omega^{2}$ in the linear one-dimensional limit. This gives

$$
L_{d p} \simeq\left(2 \omega^{2} / \omega_{p}^{2}\right) r_{b}\left(1-k^{2} r_{\beta}^{2} / 4 \gamma\right)^{-1}
$$

assuming a constant energy electron, where $L_{d p 0}=\left(2 \omega^{2} / \omega_{p}^{2}\right) r_{b}$ is the dephasing length in the absence of a betatron oscillation. Hence, increasing the betatron orbit from an amplitude of $r_{\beta 1}$ to an amplitude $r_{\beta 2}$ will increase the dephasing length by an amount $\Delta L_{d p}$, where

$$
\Delta L_{d p} / L_{d p 0} \simeq\left(k^{2} / 4 \gamma\right)\left(r_{\beta 2}^{2}-r_{\beta 1}^{2}\right)
$$

assuming $k^{2} r_{\beta}^{2} / 4 \gamma<1$. A substantial increase in the dephasing length can result when $\gamma$ is near $k^{2} r_{\beta}^{2} / 4$, however, $\gamma$ is not constant (e.g., increasing ) due to the wakefield. For high electron energies, $\gamma \gg k^{2} r_{b}^{2} / 4$, the increase in the dephasing length will be small. 
An alternative method for increasing the dephasing length and the electron energy is to introduce a region of higher density. For simplicity, consider an axial plasma density profile that is a step function in which the density increases from a value $n_{0}$ to a value $n_{1}$. As the electron is accelerated through the first (lower density) region, it outruns the wake and approaches the center of the blow-out cavity at which point the accelerating field is zero. The distance required to reach the center of the cavity defines the dephasing length. If the density is increased just prior to the electron reaching the center of the cavity, the cavity radius (proportional to the plasma wavelength) decreases, and the center of the cavity is suddenly shifted forward closer to the drive laser pulse. Hence, increasing the density can increase the dephasing length and the electron energy gain $[24,25]$. The increase in the dephasing length is $\Delta L_{d p} \simeq\left(2 \omega^{2} / \omega_{p 1}^{2}\right)\left(r_{b 0}-r_{b 1}\right)$, where the subscripts 0 and 1 refer to the values in the density regions $n_{0}$ and $n_{1}$, respectively. Since $\omega_{p} \propto n^{1 / 2}$ and $r_{b} \propto n^{-1 / 2}$,

$$
\Delta L_{d p} / L_{d p 0}=\left(n_{0} / n_{1}\right)\left[1-\left(n_{0} / n_{1}\right)^{1 / 2}\right]
$$

which is independent of the electron energy (assuming $\gamma>\omega / \omega_{p}$ ), neglects the effects of the betatron orbit, and assumes that the laser intensity $a_{0}$ is constant. Since the wakefield scales with density as $E_{z} \propto n^{1 / 2}$, the final electron energy at dephasing scales as $\Delta \gamma_{d p} / \gamma_{d p 0}=$ $\left(n_{0} / n_{1}\right)^{1 / 2}\left[1-\left(n_{0} / n_{1}\right)^{1 / 2}\right]$. Furthermore, the betatron period $\lambda_{\beta} \propto n^{-1 / 2}$ is reduced in the higher density region, which increases the number of betatron oscillations and hence the flux of the emitted radiation.

Figure 2 shows the electron orbits obtained by numerically integrating Eq. (1) for four cases: (a) a uniform density case with $n_{1}=n_{0}$, (b) a plasma with a narrow depleted region of density $n_{1}=n_{0} / 10$, (c) a plasma with a wider depleted region of density $n_{1}=0$, and (d) a plasma with a density step with $n_{1}=1.5 n_{0}$. In these two-dimensional simulations, the betatron orbit is in the $x-z$ plane with an initial amplitude $x_{i}=2 \mu \mathrm{m}$, initial momenta $p_{x_{i}}=0$ and $p_{z_{i}} / m c=10$, with $n_{0}=2 \times 10^{19} \mathrm{~cm}^{-3}$.

Simple expressions for the location of the density depleted region $z_{1}$ and the length of the depleted region $L=z_{2}-z_{1}$ that maximize the electron betatron amplitude and energy can be estimated as follows. If we assume that $F_{\|}$is negligible in the depleted region from $z_{1}$ to $z_{2}\left(0<n_{1}<n_{0}\right)$ and that the electron has a constant energy $\gamma\left(z_{1}\right)$, then the equation of motion reduces to an harmonic oscillator and the transverse motion is given by

$$
x(t)=x_{1} \cos \left(\omega_{\beta_{1}} t+\phi\right),
$$




$$
v_{x}(t)=-x_{1} \omega_{\beta_{1}} \sin \left(\omega_{\beta_{1}} t+\phi\right)
$$

where $x_{1}=\left(x^{2}+v_{x}^{2} / \omega_{\beta_{1}}^{2}\right)^{1 / 2}$ is a constant of motion corresponding to the amplitude of the betatron oscillation [29], $\omega_{\beta_{1}}=\omega_{\beta}\left(n_{1}, \gamma\left(z_{1}\right), x_{1}\right)$ is the betatron frequency in the region of density $n_{1}$, and $\phi$ is a constant phase term. In the region $\left[z_{1}: z_{2}\right]$, the amplitude $\left|x_{1}\right|$ of the betatron motion depends on the electron energy and position at $z_{1}$. It oscillates at $2 \omega_{\beta_{1}}$ in the range

$$
x_{1}= \begin{cases}x_{0}\left(n_{0} / n_{1}\right)^{1 / 2}, & \text { for } x\left(z_{1}\right)=0, \\ x_{0}, & \text { for } x\left(z_{1}\right)=x_{0},\end{cases}
$$

where $x_{0}$ is the betatron amplitude before $z_{1}$ and where we have used the relation $v_{x}\left(z_{1}\right) / \omega_{\beta_{1}}=x_{0}\left(n_{0} / n_{1}\right)^{1 / 2}$. In order to maximize $x_{1}$, the position $z_{1}$ must be chosen to have $x\left(z_{1}\right)=0$ and the length $L$ must match one quarter of a betatron period $\left(\lambda_{\beta_{1}} / 4\right)$, which gives

$$
L=\frac{\lambda_{p}\left(n_{1}\right)}{4} \sqrt{2 \gamma\left(z_{1}\right)}
$$

In Fig. 2(b), the density depletion starts at $z_{1}=190 \mu \mathrm{m}$. At that position the electron has an energy $\gamma=150$ and $x_{0}=0.6 \mu \mathrm{m}$. In agreement with the simulation, the above expressions give $x_{1}=1.9 \mu \mathrm{m}$ and $L=100 \mu \mathrm{m}$.

For the case $n_{1}=0$, the electron is in a force free region for $z \in\left[z_{1}: z_{2}\right]$. The electron motion is ballistic and the transverse amplitude is given by

$$
x_{1}=\frac{v_{x}\left(z_{1}\right)}{v_{z}\left(z_{1}\right)} L \sim \frac{a_{\beta 0}}{\gamma\left(z_{1}\right)} L
$$

where $a_{\beta 0}=a_{\beta}\left(n_{0}, \gamma\left(z_{1}\right), x_{0}\right)$. The transverse orbit $x_{1}$ is limited by the size of the cavity. If $x_{1}$ exceeds the transverse dimension of the cavity it is not trapped at $z_{2}$. This gives the condition

$$
L<\frac{v_{z}\left(z_{1}\right)}{v_{x}\left(z_{1}\right)} r_{b}\left(z_{1}\right) \sim \frac{\gamma\left(z_{1}\right)}{a_{\beta 0}} r_{b}\left(z_{1}\right),
$$

where $r_{b}\left(z_{1}\right)$ is the radius of the ion cavity at the position $z_{1}$. In the example of Fig. 2(c), the density depletion is placed at $z_{1}=416 \mu \mathrm{m}$. At that position the electron has an energy $\gamma=258$. Here, we have chosen the thickness of the depleted density slice to be $L=220 \mu \mathrm{m}$, which gives $x_{1}=4 \mu \mathrm{m}$.

In the case of the density step, the transverse orbit amplitude, within the harmonic oscillator approximation, is given by Eq. (13). In the longitudinal direction, the radius of the cavity is reduced to $r_{b}\left(n_{1}\right)$ and the condition for the electron to remain trapped in the 
cavity at the density transition is then

$$
z_{1}>v_{g} t-2 r_{b}\left(n_{1}\right)
$$

In addition to the modification of the electron orbit amplitude, a consequence of the density modulation is an energy gain with respect to the reference case of uniform density $\left(n_{1}=n_{0}\right)$. Figure 3 shows the electron energy as a function of time for the three cases that correspond to those in Fig. 2 (b)-(d). The insert of Fig. 3 shows the normalized acceleration force $F_{\|}$. For the reference case, $F_{\|}$linearly decreases as a function of time and the electron outruns the wake after $\sim 1 \mathrm{~mm}$. Its maximum energy is $\gamma_{0}=380$. In the case of the density depletion, the increase of the electron energy occurs from a modification of the dephasing length. Because of the longer path the electron has to cover, $d F_{\|} / d t$ is lowered and the time for the electron to reach the dephasing phase of the plasma wave is increased. This clearly appears on the inset of Fig. 3. As a result, the maximum electron energy is increased. For the example in Fig. 3, the electron energy becomes $\gamma_{1}=520$ in the case $n_{1}=0$ and $\gamma_{1}=440$ for $n_{1}=n_{0} / 10$. For the density step, the principle is different since betatron amplitude is not increased. Here, the reduction of the cavity size causes the electron to slip back to the accelerating phase of the wake. The accelerating force is suddenly increased at the step and exceeds $E_{0}$ owing to the higher density. For the example in Fig. 3, the maximum electron energy reaches $\gamma_{1}=520$ and then decreases from dephasing.

\section{BETATRON RADIATION FROM A SINGLE ELECTRON}

As described in Sec. II, many of the important features of betatron radiation are characterized by the betatron strength parameter $a_{\beta} \propto(\gamma n)^{1 / 2} r_{\beta}$ and the critical frequency $\omega_{c} \propto \gamma^{2} n r_{\beta}$. As discussed in Sec. III, the amplitude of the electron motion in the ion cavity is of the order of 1-2 $\mu \mathrm{m}$. This results in maximum $a_{\beta}$ parameters typically on the order $a_{\beta} \sim 10$. The modification of the electron trajectories described above, through tailoring of the density profile, allows both $a_{\beta}$ and $\omega_{c}$ to increase so as to efficiently produce betatron radiation at higher frequencies.

With the knowledge of the trajectory parameters it is then straightforward to obtain the betatron x-ray beam features. In the presence of the density modulation, the critical x-ray energy is up-shifted owing to the increase of either $r_{\beta}, \gamma$, or $n$. The maximum increase of 
the critical frequency is given by the ratio

$$
R_{\omega_{c}}=\frac{\omega_{c 1}}{\omega_{c 0}} \simeq \begin{cases}\left(\gamma_{1} / \gamma_{0}\right)^{2}\left(n_{0} / n_{1}\right)^{1 / 2}, & \text { for } 0<n_{1}<n_{0} \\ \left(\gamma_{1} / \gamma_{0}\right)^{2}\left(x_{1} / x_{0}\right) L, & \text { for } n_{1}=0 \\ \left(\gamma_{1} / \gamma_{0}\right)^{2}\left(n_{1} / n_{0}\right), & \text { for } n_{1}>n_{0},\end{cases}
$$

where $\omega_{c 1}$ and $\omega_{c 0}$ are respectively the maxima of the critical frequency along the propagation with and without a density modulation. Also, note that for the $n_{1}=0$ case, $\left(\gamma_{1} / \gamma_{0}\right)^{2} x_{1} L / x_{0} \sim\left(\gamma_{1}^{2} / \gamma_{0}^{3}\right) L a_{\beta 0} / x_{0}$. Figure 4 shows the spectral intensity, integrated over the vertical angle [Eq. (7)], for $n_{1}=n_{0}, n_{1}=n_{0} / 10, n_{1}=0$ and $n_{1}=1.5 n_{0}$. In the case $n_{1}=0$, the spectrum extends above $100 \mathrm{keV}$. Using the results of Figs. 2 and 3, which give $x_{0}=0.6 \mu \mathrm{m}, x_{1}=4 \mu \mathrm{m}, \gamma_{0}=380$ and $\gamma_{1}=520$, we obtain $R_{\omega_{c}} \simeq 12$. In the case $n_{1}=n_{0} / 10$, we have $\gamma_{0}=380, \gamma_{1}=440$ (Fig. 3), which give $R_{\omega_{c}} \simeq 4$. Finally, for the case $n_{1}=1.5 n_{0}$, the maximum energy reached by the electron is $\gamma=520$ and $R_{\omega_{c}} \simeq 2$. The analytical results are in good agreement with the numerical results.

The gain of energy and flux provided by the modification of $r_{\beta}$ and $\gamma$ is lowered by losses in the case of the density depletion. Indeed, for $n_{1}=0$ the electron is not accelerated and does not emit radiation over $L$. This is also the case for $n_{1}=n_{0} / 10$, but with $L$ shorter (for $n_{1}=n / 10$ the acceleration and radiation emitted are negligible). On the other hand, in the case of the density step there are no losses and, owing to the reduction of the betatron period for $z>z_{1}$, the electron undergoes more betatron oscillations and the flux is further increased. The most appropriate density modulation depends on the x-ray energy range and varies depending on $n_{1}$ and $L$. In our particular example, $n_{1}=0$ is suitable for x-ray energies $\gtrsim 20 \mathrm{keV}, n_{1}=n_{0} / 10$ for energies $\in[\sim 10: \sim 20] \mathrm{keV}$ and $n_{1}=1.5 n_{0}$ for energies $<10 \mathrm{keV}$.

The position $z_{1}$ of the density modulation strongly affects the radiated energy because it determines the betatron amplitude $x_{1}$ and the energy of the electron. Figure 5 shows the radiated energy $d I\left(z_{1}\right) / d \theta_{h}=\int d \omega\left(d^{2} I / d \omega d \theta_{h}\right)$ (integrated over vertical angle and frequencies) as a function $z_{1}$ for the three cases. Here, $d I\left(z_{1}\right) / d \theta_{h}$ is normalized by the maximum radiated energy obtained in the case $n_{1}=0$. On each graph, we also show $d I\left(z_{1}\right) / d \theta_{h}$ for the reference case $n_{1}=n_{0}$. In the case of the density depletion, the radiated energy oscillates with $x_{1}$ at $2 \omega_{\beta 1}$. As seen before, the modulation must be placed at $z_{1}$ satisfying $x\left(z_{1}\right)=0$. Oppositely, for $x\left(z_{1}\right)=x_{0}$, the amplitude of motion is not increased and the radiated energy is lower than the reference case because of losses. For the density step, 
the dependence is opposite. The amplitude is maximum when $x\left(z_{1}\right)=x_{0}$ and oscillates in the range $\left[x_{0} / \sqrt{1.5}: x_{0}\right]$. Therefore, the radiated energy oscillates at $2 \omega_{\beta 1}$, but since there are no losses, the flux always remains above the reference flux. For all cases, the radiated energy globally decreases with $z_{1}$ because the fraction of the propagation length over which the electron benefits from a larger $r_{\beta}$ and $\gamma$ is shortened.

The angular distribution of the betatron radiation in the horizontal plane (the plane containing the betatron orbit) depends on the betatron amplitude, is characterized by the angle $\theta_{h} \simeq a_{\beta} / \gamma$, and is hence affected by the density modulation. The consequence of the larger betatron amplitude is an increase in the x-ray beam divergence in the plane of the electron motion. The increase of divergence compared to the reference case is given by the ratio

$$
R_{\theta_{h}}= \begin{cases}\left(\gamma_{0} / \gamma_{1}\right)^{1 / 2}\left(x_{1} / x_{0}\right), & \text { for the depletion case } \\ \left(\gamma_{0} / \gamma_{1}\right)^{1 / 2}\left(n_{1} / n_{0}\right)^{1 / 2}, & \text { for the step case }\end{cases}
$$

where $\gamma_{1}$ and $\gamma_{0}$ are respectively the maxima of the electron energy with and without mod-

ulation. For $n_{1}=0, n_{1}=n_{0} / 10$ and $n_{1}=1.5 n_{0}$, we obtain $R_{\theta}=5.7, R_{\theta}=3$ and $R_{\theta}=1.3$. Figure 6 represents the angular profile of radiation produced by the electron trajectories of Fig. 2. The intensity represents the total radiated energy $d I / d \Omega=\int d \omega d^{2} I / d \omega d \Omega$ per unit solid angle $d \Omega$. Even considering the increase of the divergence, the radiated energy at the angle of maximum emission is enhanced.

\section{BETATRON RADIATION FROM AN ELECTRON BUNCH}

In the case of an electron beam composed of many electrons with different energies, transverse and longitudinal positions, and divergences, there are no phase relations between the electrons. Therefore, according to Fig. 5, a fraction of the electrons will benefit from the effect of the density modulation to produce more betatron radiation, whereas others will not, depending on the phase of the betatron oscillation of a given electron as it enters the depletion region. Here we consider $\sim 500$ electrons initially distributed within a cylindrical spatial region with initial radii $r_{i} \leq 2 \mu \mathrm{m}$, initial longitudinal positions $-r_{b} \leq z_{i} \leq-r_{b} / 4$, and with initial transverse momenta $-5 \leq p_{x i} / m c \leq 5$. The trajectories of all the electrons are shown in Fig. 7 for the same parameters as Fig. 2.

The phase mixing initially introduced prevents coherent betatron oscillations of the elec- 
trons, as shown on Fig. 7(a), which plots the electron trajectories for the case of no density modulation. In addition, the spread in the initial longitudinal position results in a broad electron distribution. The trajectories for the $n_{1}=n_{0} / 10, n_{1}=0$ and $n_{1}=1.5 n_{0}$ cases are shown in Figs. 7(b), (c) and (d), respectively. It is clear that the electrons have a range of betatron amplitudes $x_{1}$ as they enter the density transition.

The corresponding spectra of the radiation emitted by the electron bunches are shown in Fig. 8. They have been normalized so they can be compared to Fig. 4, which is equivalent to the case of a fully coherent oscillation of all the electrons. The difference observed between Figs. 4 and 8 can be explained using the results of Fig. 5. In the cases of the density depletion, the radiated energy from a single electron varies rapidly with $z_{1}$ (which determines the phase of the betatron oscillation as it enters the density transition) and because of the large phase variation of the electrons in the bunch at $z_{1}$, it is lowered compared to Fig. 4 . In the case of the density step, the radiated energy only slightly varies with $z_{1}$ and it remains very similar to Fig. 4. The x-ray spectra that can be obtained will depend on the electron bunch parameters, and will vary between Figs. 4 and 7.

\section{DISCUSSION}

Methods have been investigated for enhancing both the flux and photon energy of betatron radiation by modifying the longitudinal plasma density profile while holding the laser parameters constant. Two specific types of density profiles were examined. The first is to introduce a region of depleted density in an otherwise initially uniform plasma. The second is a step function profile, wherein the density is increased in the latter portion of the plasma. The density depletion region, if positioned properly in the plasma, increases the betatron amplitude of significant fraction of the accelerated electrons. The increased betatron amplitude also implies a decreased axial velocity and hence an increased dephasing length and a increased energy gain. Thus a depletion region can increase both $r_{\beta}$ and $\gamma$, and hence $\omega_{c}$. A step profile with increased density decreases the plasma wavelength, thereby increases the dephasing length and the energy gain. Thus a density step can increase both $n$ and $\gamma$, and hence $\omega_{c}$.

Numerical studies were carried out to determine the motion of test particles in analytically specified fields for cavitated wakes including the effects of density tailoring. The cases of a 
single electron and of an electron beam were investigated. The type of density modulation depends on the energy range desired and can be either a density dip or step. Several potentially significant effects were not modelled. In particular, laser evolution was not included in this study. For example, the propagation of the laser through the density depleted region has not been treated. If the plasma density is too low, guiding of the laser pulse may not occur (through relativistic self-focusing and/or channel guiding). In that case, the laser diffracts and if the laser intensity is too low at $z_{2}$, the cavitated wake does not immediately reform and loss of accelerated electrons becomes significant. It is therefore necessary to have $L$ smaller than the Raleigh length. Similarly, laser pulse depletion has not been modelled. In the highly nonlinear regime, the pump depletion length may not significantly exceed the dephasing length, which will limit the applicability of density tailoring to enhance the energy gain beyond the dephasing limit in a uniform plasma. Inclusion of these effects requires self-consistent simulation of laser propagation, wakefield generation, and electron acceleration.

In this article, we have only discussed the two simple configurations of density tailoring: The use of a single depleted region or the use of a single density step. Other configurations may be more efficient for enhancing the betatron emission, such as the use of multiple depletion regions or the use of a density profile which is continuously increasing. For example, consider the use of two depletion regions. First, in the case $n_{1}=0$, the use of two depletion regions can mitigate limitations due to the laser diffraction and electron loss. The sum of the lengths of two dips is less than that of a single region alone, since $v_{x}$ is higher after the first region. Second, for $n_{1}=n_{0} / 10$, the transverse oscillation amplitude can be increased in successive modulations up the the maximum value of $r_{\beta}$. This can significantly increase the x-ray flux and energy.

Density tailoring, through a variety of possible configurations, can be used to control the the electron orbits and produced more efficiently betatron radiation. For fixed drive laser parameters, it is found that the betatron radiation spectra can be shifted from the $10 \mathrm{keV}$ range to the $100 \mathrm{keV}$ range. In the $100 \mathrm{keV}$ energy range, the betatron source could become a pioneering and promising tool for the applications requiring energetic, ultrashort pulse and micrometer source size x-ray radiation. 


\section{Acknowledgments}

This work was supported in part by the National Science Foundation Grant No. 0614001 and by the Director, Office of Science, Office of High Energy Physics, of the U.S. Department of Energy under Contract No. DE-AC02-05CH11231.

[1] Esarey, E., P. Sprangle, J. Krall, and A. Ting, IEEE Trans. Plasma Sci. PS-24, 252-288 (1996).

[2] D. Umstadter, J. Phys. D, 36, R151 (2003).

[3] C. Joshi, Phys. Plasmas 14, 055501 (2007).

[4] A. Pukhov and J- Meyer-ter-vehn, Appl phys. B:Lasers Opt. bf 74, 355 (2002).

[5] S. P. D. Mangles, A. G. R. Thomas, M. C. Kaluza, O. Lundh, F. Lindau, A. Persson, F. S. Tsung, Z. Najmudin, W. B. Mori, C.-G. Wahlstrom, and K. Krushelnick, Phys. Rev. Lett. 96, 215001 (2006).

[6] W. Lu, C. Huang, M. Zhou, M. Tzoufras, F. S. Tsung, W. B. Mori, and T. Katsouleas, Phys. Plasmas 13, 056709 (2006).

[7] S. P. D. Mangles, C. D. Murphy, Z. Najmudin, A. G. R. Thomas, J. L. Collier, A. E. Dangor, E. J. Divall, P. S. Foster, J. G. Gallacher, C. J. Hooker, D. A. Jaroszynski, A. J. Langley, et al., 2004, Nature 431, 535 (2004).

[8] C.G.R. Geddes, Cs. Toth, J. van Tilborg, E.H. Esarey, C.B. Schroeder, D. Bruhwiler, C. Nieter, J. Cary, and W.P. Leemans, Nature 431, 538 (2004).

[9] Faure, J., Y. Glinec, A. Pukhov, S. Kiselev, S. Gordienko, E. Lefebvre, J.-P. Rousseau, F. Burgy, and V. Malka, 2004, Nature 431, 541 (2004).

[10] Faure, J., C. Rechatin, A. Norlin, A. Lifschitz, Y. Glinec, and V. Malka, Nature 444, 737 (2006).

[11] W.P. Leemans, B. Nagler, A.J. Gonsalves, C. Toth, K. Nakamura, C.G.R Geddes, E. Esarey, C.B. Schroeder, S. Hooker, Nature Phys. 2696 (2006).

[12] G. Mourou, T. Tajima, S. Bulanov, Rev. Mod. Phys., 78, 309 (2006).

[13] E. Esarey, B.A. Shadwick, P. Catravas, and W.P. Leemans, Phys. Rev. E 65, 056505 (2002).

[14] Shuoqin Wang et al., Phys. Rev. Lett. 88, 135004 (2002) 
[15] I. Kosyukov, A. Pukhov, S. Kieselev, Phys. Plasmas 10, 4818 (2003).

[16] S. Kiselev, A. Pukhov, I. Kostyukov, Phys. Rev. Lett 93, 13, 135004 (2004).

[17] A.Rousse, K.Ta Phuoc, R. Shah, A. Pukhov, E. Lefebvre, V. Malka, S. Kieselev, F. Burgy, J-P. Rousseau, D. Umstadter and D. Hulin, Phys. Rev. Lett. 93, 13, 135005 (2004).

[18] W.P. Leemans, E. Esarey, J. van Tilborg, P.A. Michel, C.B. Schroeder, Cs. Toth, C.G.R. Geddes, and B.A. Shadwick, IEEE Trans. Plasma Sci. 30, 8-22 (2005).

[19] P. Michel, C.B. Schroeder, B.A. Shadwick, E. Esarey, and W.P. Leemans, Phys. Rev. E 74, 026501 (2006).

[20] D. K. Johnson et al. Phys. Rev. Lett. 97, 175003 (2006)

[21] K. Ta Phuoc, S. Corde, R. Shah, F. Albert, R. Fitour, J-P. Rousseau, F. Burgy, B. Mercier, and A. Rousse, Phys. Rev. Lett., 97, 225002 (2006).

[22] K. Ta Phuoc, R. Fitour, D-E. Kim et al., Phys. Plasmas, 14, 1 (2007).

[23] Handbook of Accelerator Physics and Engineering, edited by A. W. Chao and M. Tigner (World Scientific, Singapore, 1999).

[24] T. Katsouleas, Phys. Rev. A 33, 2056 (1986).

[25] Sprangle, P., B. Hafizi, J. R. Penano, R. F. Hubbard, A. Ting, C. I. Moore, D. F. Gordon, A. Zigler, D. Kaganovich, and T. M. Antonsen, Jr., Phys. Rev. E 63, 056405 (2001).

[26] Mechanical modulation

[27] C.-H. Pai, S.-Y. Huang, C.-C. Kuo, M.-W. Lin, J. Wang, S.-Y. Chen, C.-H. Lee and J.-Y. Lin, Phys. Plasma 12, 070707 (2005).

[28] C.-L. Chang, C.-T. Hsieh, Y.-C. Ho, Y.-S. Chen, J.-Y. Lin, J. Wang and S.-Y. Chen, Phys. Rev. E 75, 036402 (2007).

[29] N. Kumar and V. K. Tripathi, Europhys. Lett. 75, 26026 (2006).

[30] Applications 
Figure 1: Schematic of the density modulations considered. The density varies along the direction of the laser. The initial density is $n_{0}$. In the case of a density depletion, $n_{1}<n_{0}$ whereas $n_{1}>n_{0}$ in the case of the step.

Figure 2: Electron orbits for the cases $n_{1}=n_{0}(\mathrm{~A}), n_{1}=n_{0} / 10(\mathrm{~B})$ and $n_{1}=0(\mathrm{C})$, $n_{1}=1.5 \times n_{0}(\mathrm{D})$. The initial conditions are $x_{i}=2 \mu \mathrm{m}, p_{x_{i}}=0, p_{z_{i}} / m c=10, n_{0}=2 \times 10^{19}$ $\mathrm{cm}^{-3}$ and $a_{0}=2$.

Figure 3: Electron energy as a function of time for the trajectories of Figure 2. The insert represents the normalized acceleration force $F_{\|}$.

Figure 4: Spectral intensity, integrated over the angles, for $n_{1}=n_{0}, n_{1}=n_{0} / 10, n_{1}=0$ and $n_{1}=1.5 \times n_{0}$. The condition are the same as Figure 2 .

Figure 5: Normalized total radiated x-ray energy as a function of the position of the modulation $z_{1}$ for $n_{1}=n_{0} / 10, n_{1}=0$ and $n_{1}=1.5 \times n_{0}$. The line on each figure represents the energy in the reference case $n_{1}=n_{0}$.

Figure 6: Spatial distribution of the radiation integrated over the energy for the electron orbits of Figure 2. 

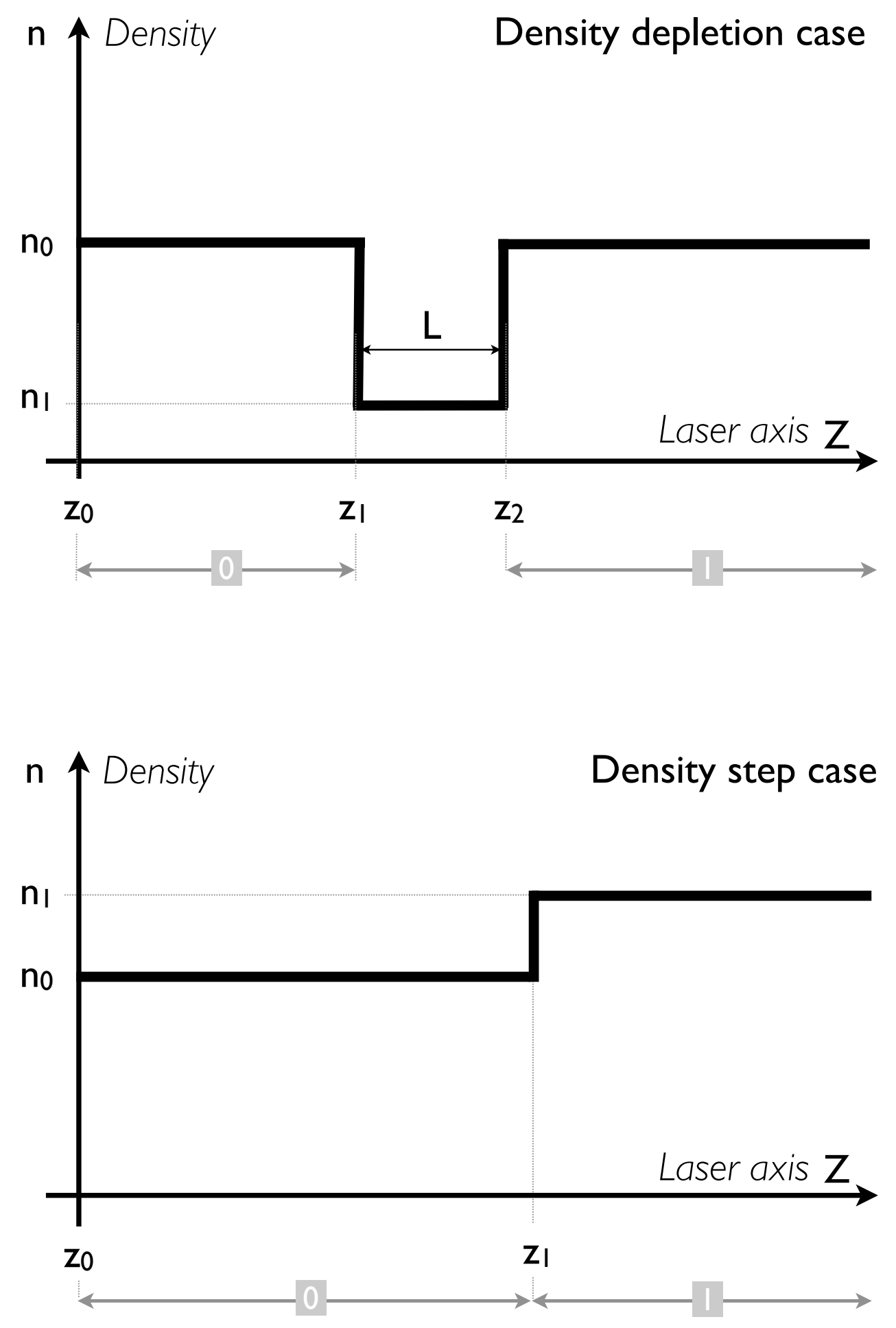

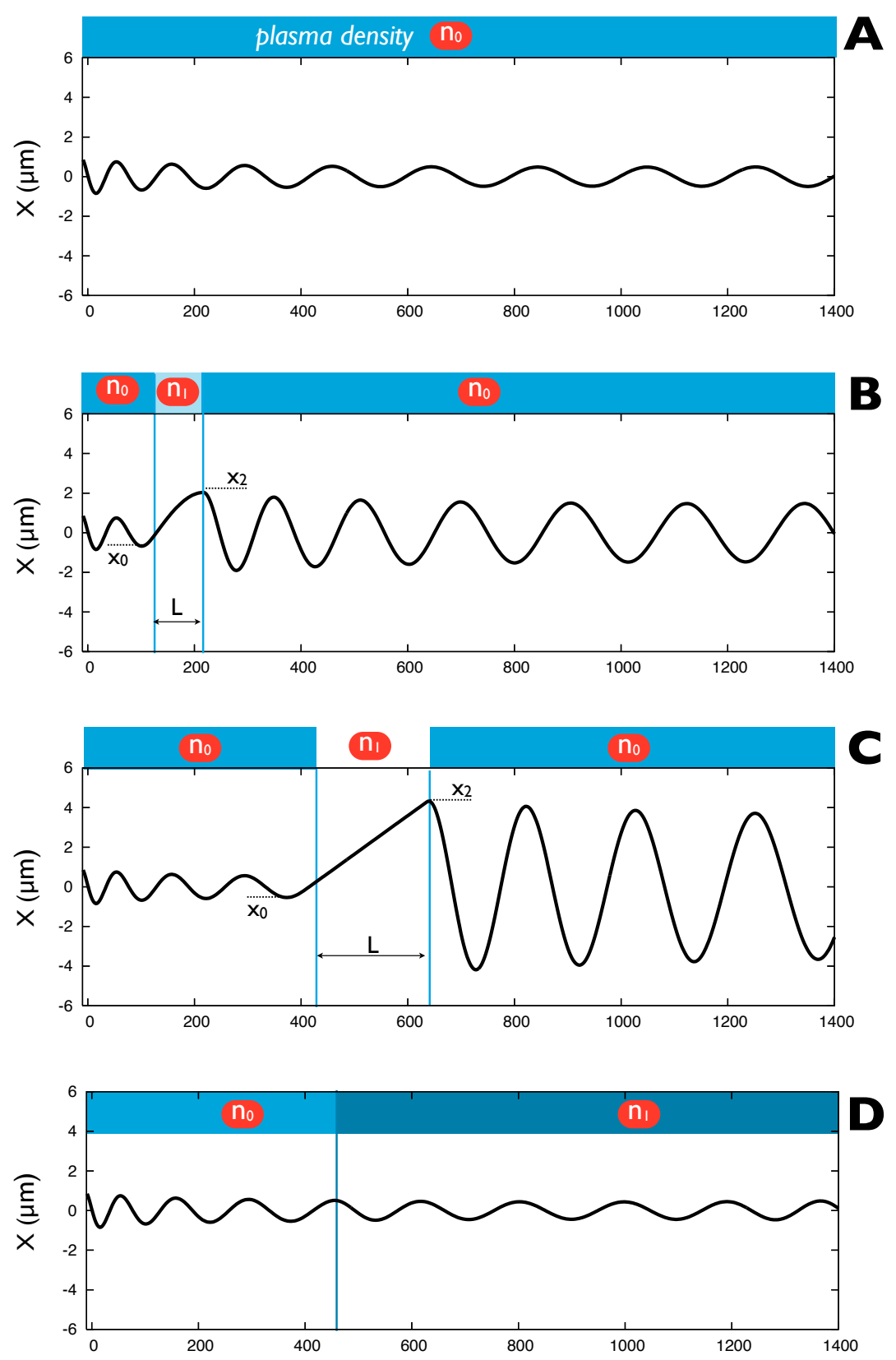

$\mathrm{Z}(\mu \mathrm{m})$ 


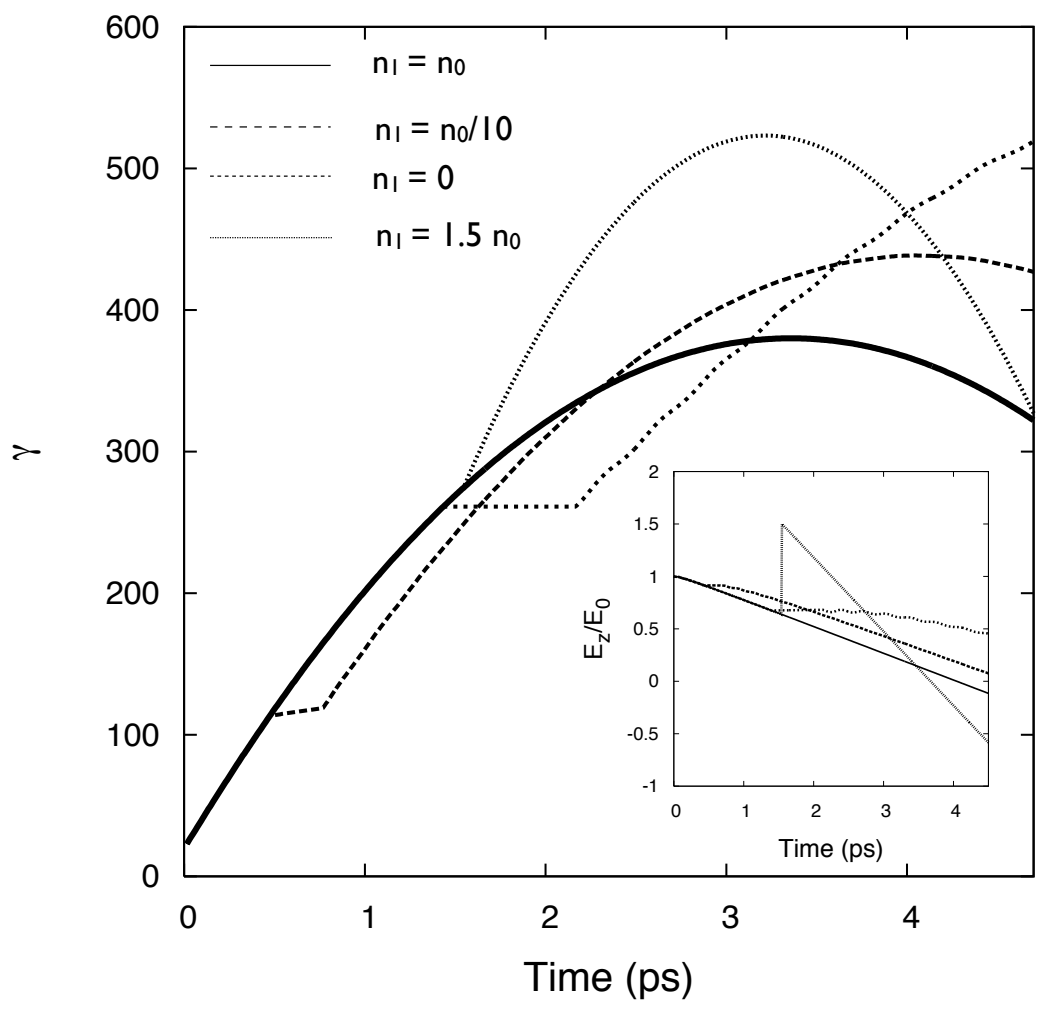




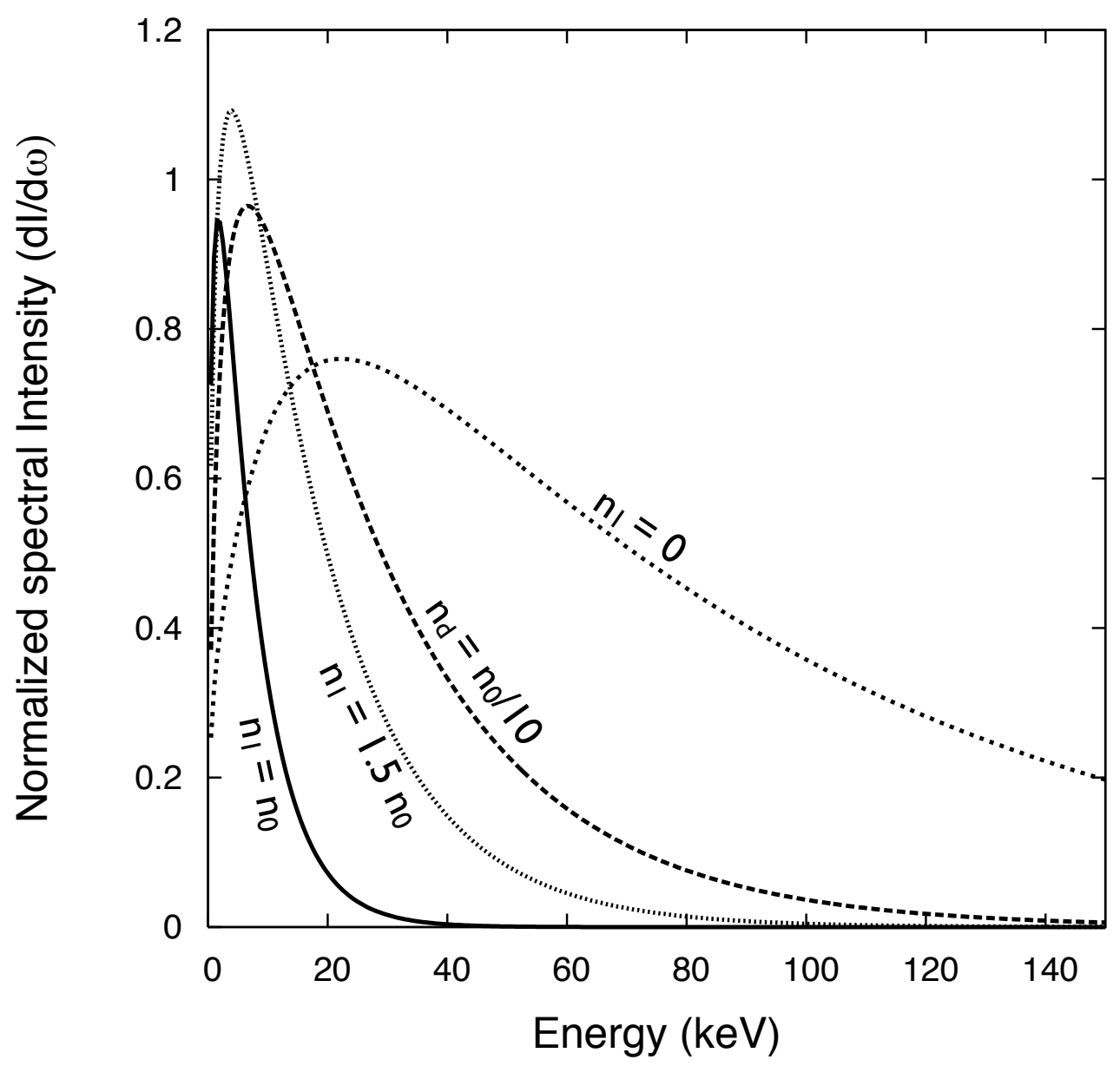



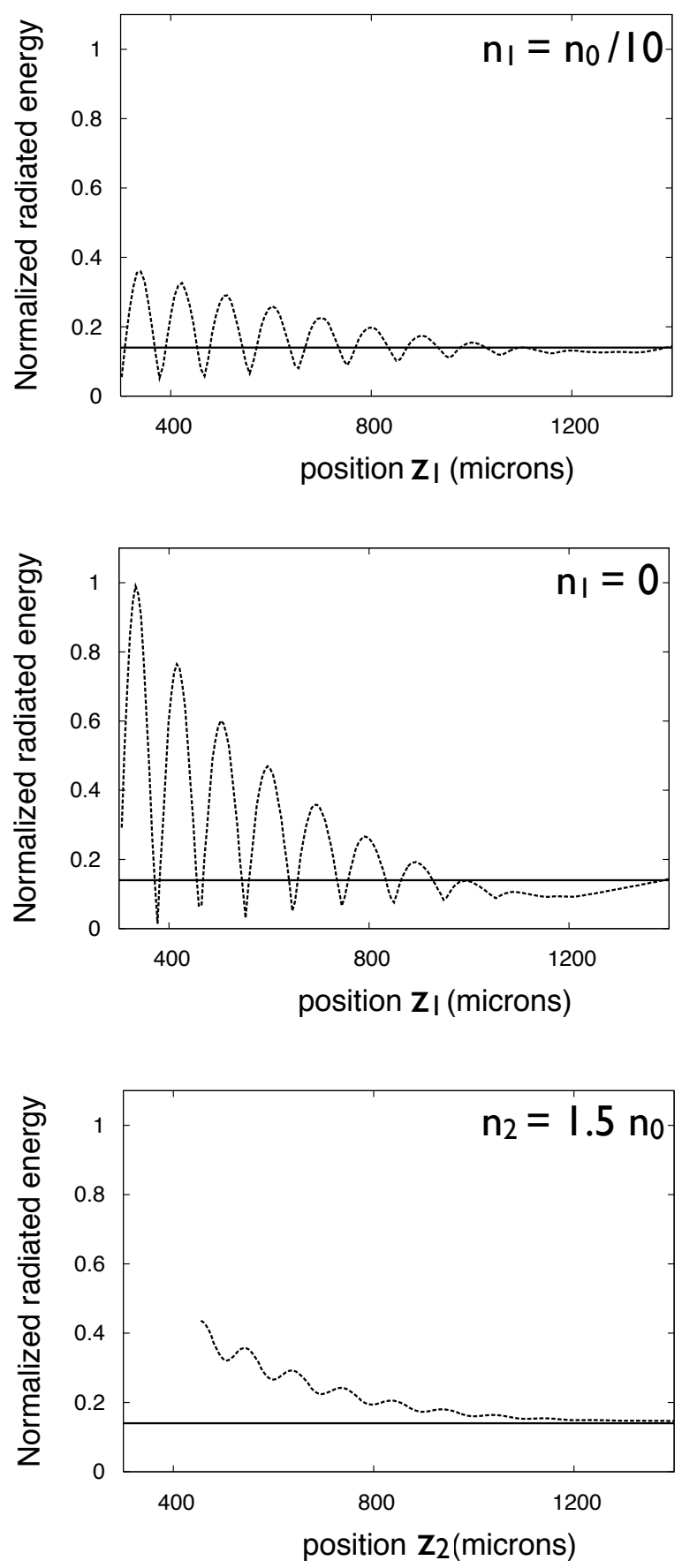


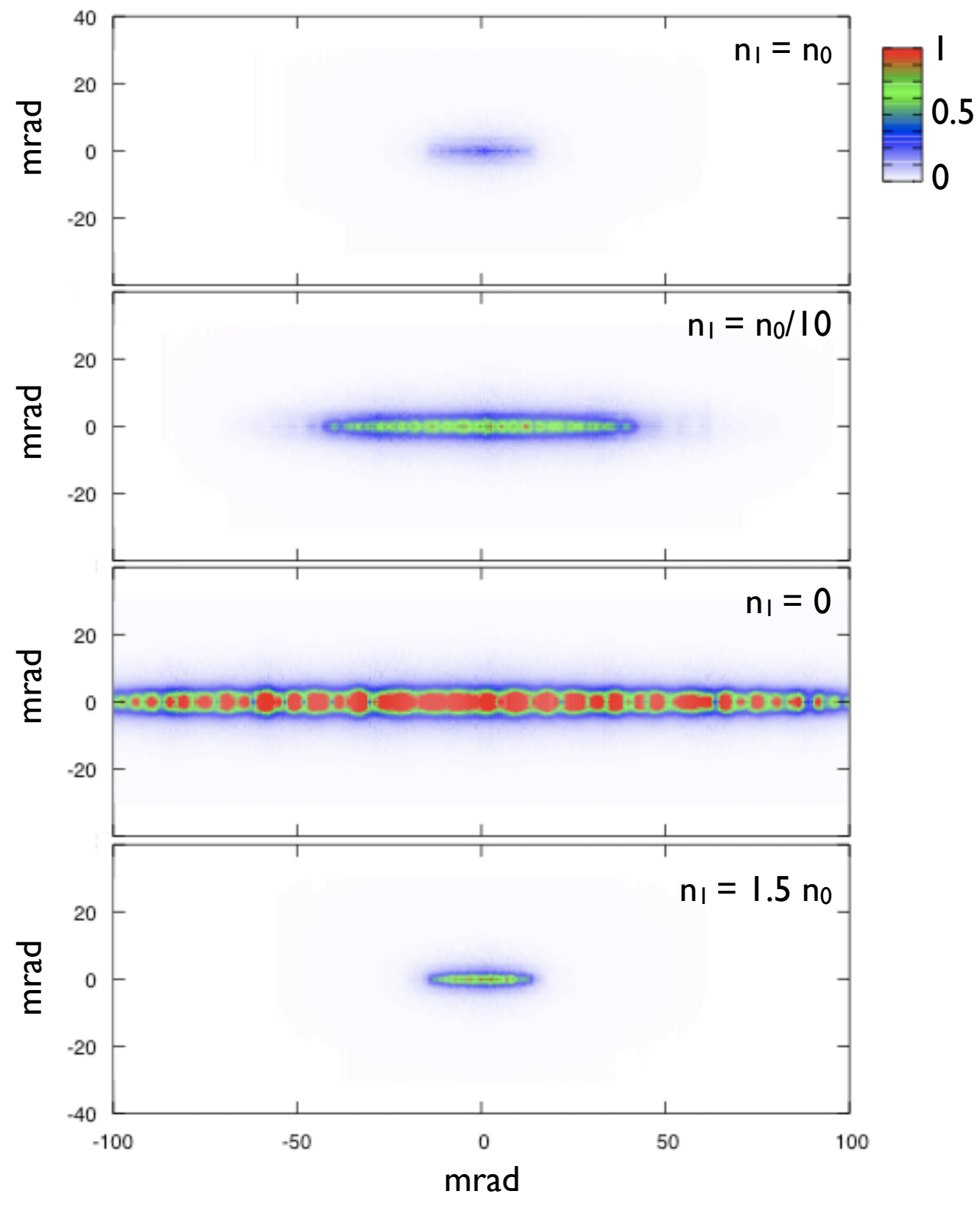



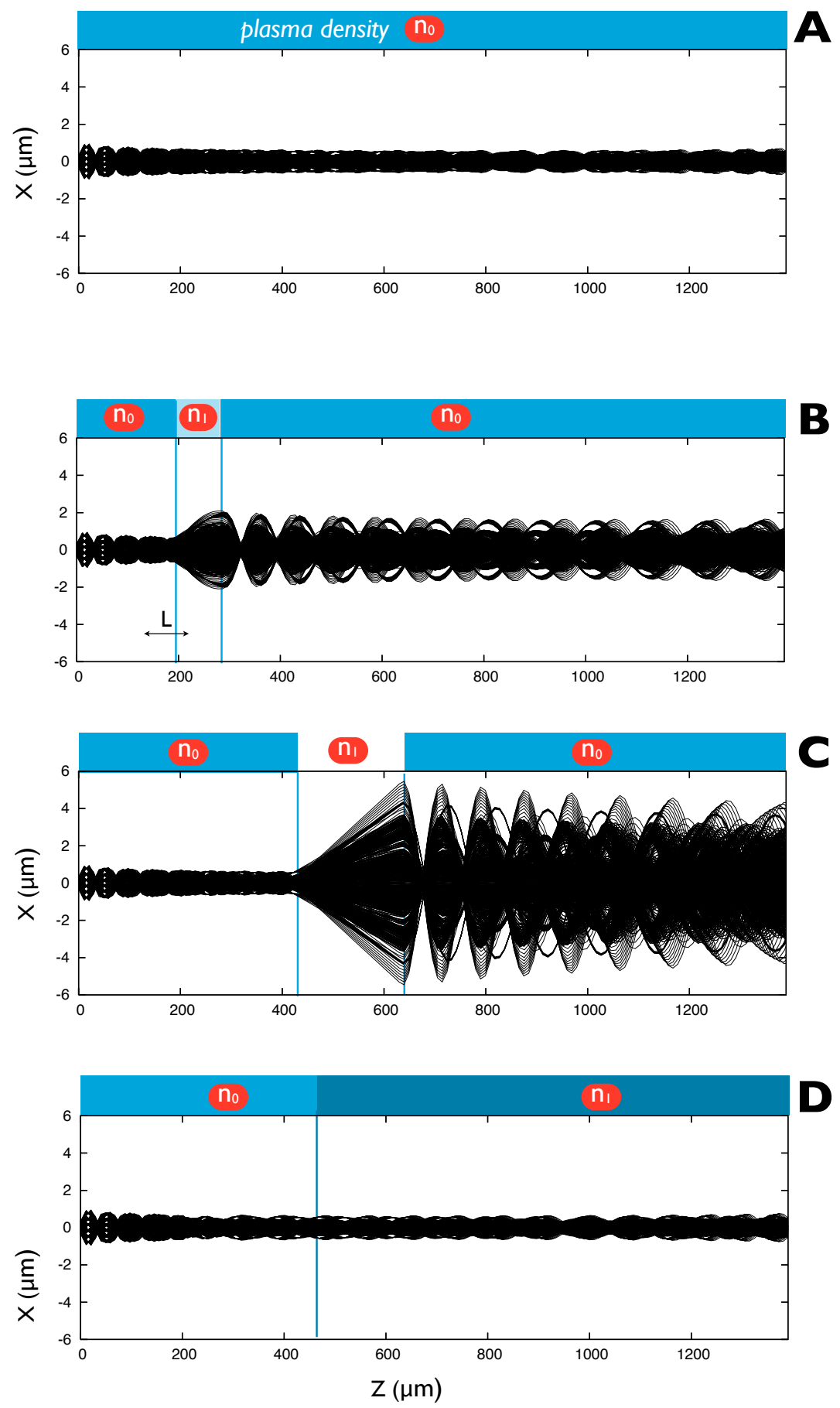


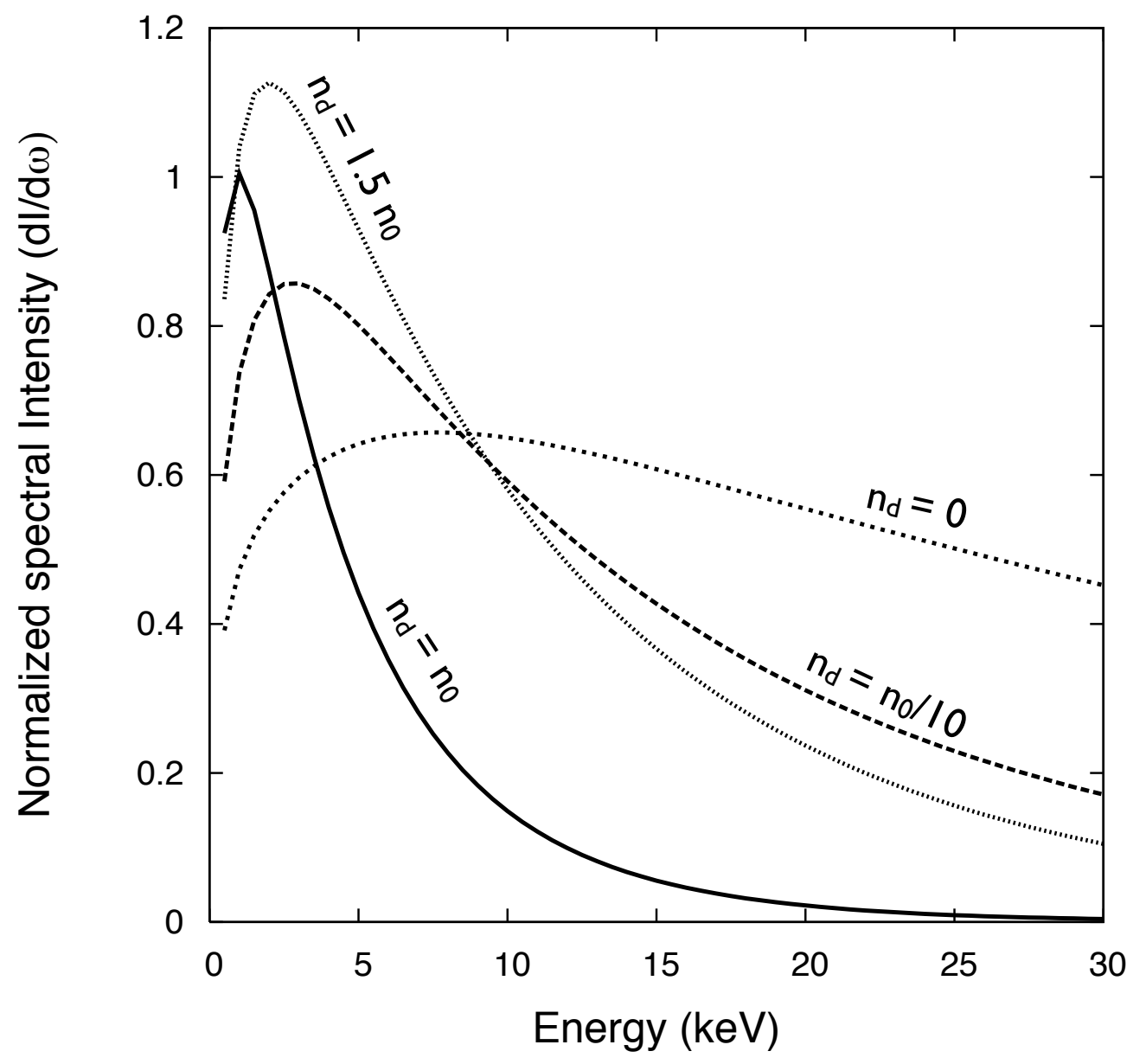

